\title{
Spontaneous Intracerebral Hemorrhage: Epidemiology, Clinical Profile and Short-Term Outcome in a Tertiary Hospital in Sub-Saharan Africa
}

\author{
Jacques Doumbe ${ }^{1}$, Khadidja Abdouramani' ${ }^{1}$, Daniel Massi Gamss ${ }^{1,2}$, Chia Mark Ayeah ${ }^{3}$, \\ Caroline Kenmegne ${ }^{4}$, Yacouba Njankouo Mapoure ${ }^{1,4^{*}}$ (]) \\ ${ }^{1}$ Department of Clinical Sciences, University of Douala, Douala, Cameroon \\ ${ }^{2}$ Department of Epidemiology, School of Public Health, University of Ghana, Accra, Ghana \\ ${ }^{3}$ Department of Clinical Sciences, University of Buea, Buea, Cameroon \\ ${ }^{4}$ Neurological Unit, Douala General Hospital, Douala, Cameroon \\ Email: dojana2003@yahoo.fr, kdikessel@gmail.com,danny.gamsmassi@gmail.com, ayeahmarkchiatoh@yahoo.com, \\ jemmycaro@yahoo.fr, *mapoureyacouba@gmail.com
}

How to cite this paper: Doumbe, J., Abdouramani, K., Gams, D.M., Ayeah, C.M., Kenmegne, C. and Mapoure, Y.N. (2020) Spontaneous Intracerebral Hemorrhage: Epidemiology, Clinical Profile and ShortTerm Outcome in a Tertiary Hospital in Sub-Saharan Africa. World Journal of Neuroscience, 10, 141-154.

https://doi.org/10.4236/wins.2020.103016

Received: May 17, 2020

Accepted: August 11, 2020

Published: August 14, 2020

Copyright ( 2020 by author(s) and Scientific Research Publishing Inc. This work is licensed under the Creative Commons Attribution International License (CC BY 4.0).

http://creativecommons.org/licenses/by/4.0/ (c) (i) Open Access

\begin{abstract}
Background: Data on spontaneous intracerebral haemorrhagic (SICH) are scarce in Africa. Our objectives were to determine the prevalence of $\mathrm{SICH}$, describe the clinical profile, aetiology and evaluate the prognosis (fatality case, functional outcome) of patients in a tertiary health care hospital in Cameroon. Methods: This was a hospital-based retrospective cohort which included patients with SICH and followed up for 6 months after stroke. Subarachnoid haemorrhage, cerebral venous thrombosis with bleeding or bleeding related with ischemic or brain tumour were excluded. Predictive factors were obtained using multiple logistic regression and survival by Kaplan Meier method. Results: The prevalence of SICH was 37\% with male predominance (64.0\%), a mean age of $55.6 \pm 11.8$ years. Deep coma was found in $30.3 \%$ on admission. The basal ganglion was the most frequent location of haemorrhage (85.1\%) while intraventricular blood effusion, mass effect, cerebral oedema and herniation occurred in $31.4 \%, 25.7 \%, 8.8 \%$ and $5.0 \%$ respectively. Hypertension (57.5\%) was the most common aetiology. The mean length of hospitalization was $9.0 \pm 7.7$ days and chest infection (30.7\%) was the most frequent complication. The cumulative case fatality rate after 24 hours (day 1), during admission, month 1 and month 3 was 9.6\%, 39.9\%, $46.0 \%, 59.8 \%$ respectively. On multivariate analysis, GCS $<9$ [OR $(95 \% \mathrm{CI})=$ 3.538 (1.086 - 11.526), $\mathrm{p}=0.036$ ] and NIHSS $15-24$ [OR $(95 \% \mathrm{CI})=7.498$ (1.306 - 43.029), $\mathrm{p}=0.024]$ were independent predictors of in-hospital mor-
\end{abstract}


tality while mass effect $[\mathrm{OR}(95 \% \mathrm{CI})=3.563(1.217-10.432), \mathrm{p}=0.020]$ and hyperthermia [OR $(95 \% \mathrm{CI})=4.645(1.341-16.085), \mathrm{p}=0.015$ ] predict poor functional outcome. Six-month survival was $37.8 \%$. Conclusion: About one-third of stroke patient were haemorrhagic. Hypertension is the leading CVRF and aetiology of spontaneous ICH. About 1 over 2 patients with SICH would die within 3 months while $50 \%$ of survivors would have a poor functional outcome at $6^{\text {th }}$ month.

\section{Keywords}

Spontaneous Intracerebral Haemorrhage, Epidemiology, Prognosis, Africa

\section{Introduction}

Stroke is a leading cause of mortality and long term disability worldwide [1] [2]. Globally, stroke comprises approximately 10 percent of all deaths and causes 5.5 million deaths each year, with 44 million disability-adjusted life-years (DALYs) lost [3] [4]. In 2010, there were 16.9 million strokes worldwide, of which 70 percent occurred in low and middle-income countries; this trend is expected to increase over the next 20 years [3] [5]. SICH is twice as frequent in low-to-middle income countries (LMICs) compared to high-income countries [6]. Presently, LMICs account for more than 85 percent of the global stroke mortality [7]. The rate of mortality and poor functional recovery amongst patients with SICH is higher compared to those with ischemic stroke [8]. Over the last decade, there has been an increase in stroke admission and case-fatality in Cameroon [9]. In Cameroon, SICH accounts for $48 \%$ of stroke cases and hypertension (HTN) is the predominant cerebrovascular risk factor [10]. Over the last 3 decades, the overall incidence of SICH has remained unchanged, but its regional incidence varies by race, sex, season and geographical location [11]. Understanding the epidemiology of SICH will help influence treatment decisions towards optimal management of SICH and contribute to the limited available data on ICH in sub-Saharan Africa. The aim of this study was to determine the epidemiology, describe the clinical profile, aetiology, and evaluate the prognosis (fatality case, functional outcome) of patients in a tertiary health care hospital in Cameroon.

\section{Methods}

\section{Patients and study design}

We carried out a hospital-based retrospective cohort study in a tertiary care hospital which included consenting patients admitted for SICH in the Neurology Unit and the Intensive Care Unit (ICU) of the Douala General Hospital (DGH) from January 2010 to December 2015. Patients aged 15 years and above who were admitted for confirmed SICH within 7 days of onset of symptoms were included in our study. Patients with incomplete files (unconfirmed stroke), subarachnoid haemorrhage, traumatic ICH, haemorrhagic cerebral tumours, acute 
ischemic stroke, acute ischemic stroke with haemorrhagic transformation and cerebral venous thrombosis were excluded.

\section{Data collection and patient management}

For every patient with SICH, sociodemographic data, cerebrovascular risk factors (CVRFs), clinical and paraclinical profile were collected. The following definitions and standard operating techniques were used to identify risk factors for stroke in each subject: Hypertension was defined as patient with medical history of hypertension, treated or not and patient with persistent high blood pressure $>140 / 90 \mathrm{mmHg}$ after stroke. Diabetes Mellitus was defined as patient with medical history of diabetes, treated or not, random serum glucose $\geq 2 \mathrm{~g} / \mathrm{L}$ or venous fasting glucose test $\geq 1.26 \mathrm{~g} / \mathrm{L}$. Dyslipidemia was defined as patient with medical history of dyslipidemia or Total cholesterol $>2 \mathrm{~g} / \mathrm{L}$ or Low-density lipoprotein $>1 \mathrm{~g} / \mathrm{L}$ or High-density lipoprotein $<0.40 \mathrm{~g} / \mathrm{L}$ in males or $<0.50 \mathrm{~g} / \mathrm{L}$ in females, triglycerides $>1.50 \mathrm{~g} / \mathrm{L}$. On admission, blood samples were collected from all patients during the first 24 hours of admission to do complete metabolic panels (glycaemia, urea, creatinine, uric acid, serum electrolytes) and lipid profile using the Cobas 311 autoanalyzers. A full blood count with platelet counts, prothrombin time, cephaline-kaolin time, C-reactive protein (CRP), erythrocyte sedimentation rate (ESR), and HIV serology were done. Other tests were prescribed if required by the patient's conditions: chest X-ray, urine culture, hemoculture, and thick blood film to check for plasmodium falciparum. Neurological assessment was done by a neurologist or intensive care specialist while the interpretation of CT scan films was done by both radiologist and neurologist. On admission, stroke severity was assessed using the National Institute of Health Stroke Scale (NIHSS) and the Glasgow coma scale for patients admitted in the ICU while the functional outcome was assessed using the modified Rankin score (mRS). Follow up was done daily for clinical evaluation and complications were noted. Oxygen was administrated if ambient oxygen saturation was less than $94 \%$. Paracetamol was used $1 \mathrm{~g}$ every six hours if temperatures superior to $37.5^{\circ} \mathrm{C}$ were noted. Prevention of deep venous thrombosis and stress ulcers was done using prophylactic dose of enoxaparin $(40 \mathrm{mg})$ and omeprazole $(20 \mathrm{mg})$, respectively. An insulin protocol was set up when capillary glycaemia was above 1.4 $\mathrm{g} / \mathrm{L}$. Concerning the blood pressure, nicardipine was given intravenously with electric syringe in case of high blood pressure with a target of 140 to $160 \mathrm{mmHg}$ for systolic blood pressure in haemorrhagic stroke. In the absence of $20 \%$ mannitol, furosemide was administered to patients with evidence of cerebral oedema. Nasogastric tubes were inserted for feeding of patients with deglutition problems. Antibiotics and artemether were used for bacterial infection and malaria, respectively. Stroke death and cause of death during admission and within 6 months post stroke was also recorded. After the initial stroke discharge, patients were booked for follow up visits at 1, 3 and 6 months post SICH. For every follow visit, the stroke severity and functional outcome of stroke survivors was reassessed using the NIHSS and mRS. Poor functional outcome was considered in 
patients with $\mathrm{mRS}>2$ within the first 6 months post stoke discharge while good functional outcome was considered in patients who are alive within the first 6 months post stroke and with $\mathrm{mRS} \leq 2$. The carers of patients who did not come for routine follow up visits were contacted using cells phone for vitality status of the stroke patients.

\section{Statistical analysis}

Statistical analysis was done using the Statistical Package for Social Sciences (SPSS) Standard version, Release 20.0 (IBM Inc. 2012). Mean and standard deviation (SD) of all continuous data are reported. Chi Square test and Independent Samples t-test were used to test for the factors associated with stroke mortality and functional recovery. Independent predictors for mortality and functional recovery were determined using the cox regression model analysis. Survival analysis was performed using Kaplan Meier analysis. Level of significance was considered 0.05 (two-sided).

\section{Ethical consideration}

This study was approved by the Institutional Ethics Committee of Research on Human Health of the University of Douala and the authorities of the DGH. Confidentiality, anonymity and privacy of all patients' medical records were guaranteed at all levels of this study.

\section{Results}

\section{Prevalence of $\mathrm{ICH}$}

In this study, we registered a total of 704 confirmed cases of acute stroke, with ICH representing 37.1\% (261/704), as seen in Figure 1.

\section{Sociodemographic characteristics}

Of the 261 patients with SICH, 167 (64\%) were males with a sex ratio of 1.77. The age ranged from 23 to 95 years with a mean age of $55.6 \pm 11.8$ years and patients with $\mathrm{ICH}$ aged > 55 years were the most frequent group (50.6\%). The

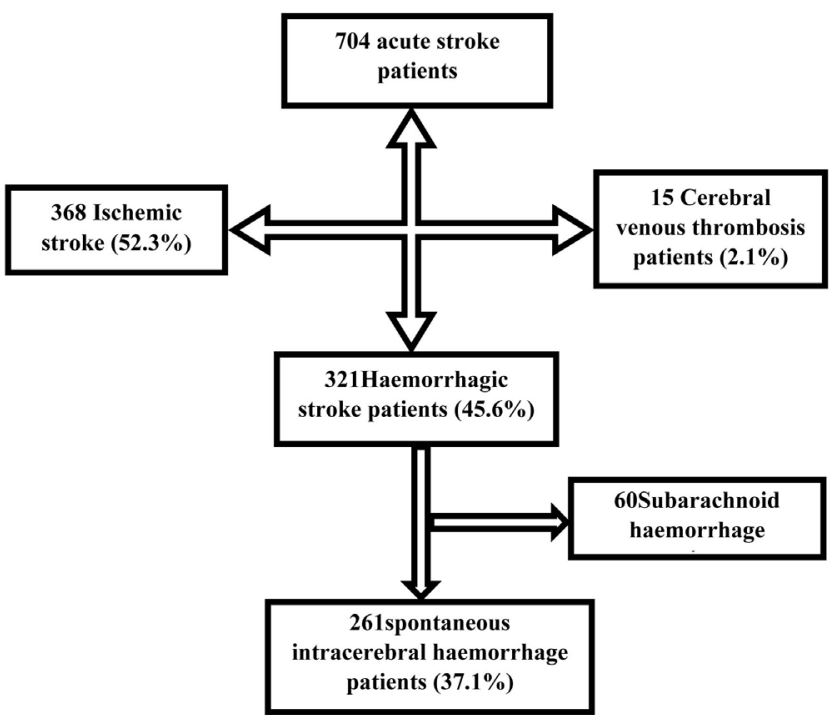

Figure 1. Flow chart for patients inclusion. 
mean age of female patients with SICH was $57.3 \pm 12.3$ years compared to $54.6 \pm$ 11.4 years amongst the male patients and there was no significant difference (Table 1).

\section{Cerebrovascular risk factors (CVRF)}

At the time of $\mathrm{SICH}$, the most frequent CVRF was hypertension representing $73.2 \%(191 / 261)$ of the patients with ICH. Obesity, diabetes mellitus, alcohol abuse, smoking and a history of previous stroke were amongst the five most frequent CVRF after hypertension (Table 1).

Time delay from onset of symptoms to stroke diagnosis

The mean time delay in initial consultation at any health facility from onset of stroke symptoms was $16.70 \pm 41.88$ hours while the mean time delay in consultation at the stroke unit of the DGH from onset of stroke symptoms was $52.72 \pm$ 84.09 hours. Upon arrival at the stroke unit of the DGH, the mean time delay in obtaining CT scan results from time of stroke symptoms onset was $47.20 \pm 64.82$ hours. Only 64 (24.5\%) patients with SICH consulted at the stroke unit within 6 hours of onset of symptoms (Table 2).

Clinical presentation, site of haemorrhage and associated lesions on imagery

Table 1. Sociodemographic characteristics and cerebrovascular risk factors amongst ICH patients.

\begin{tabular}{|c|c|}
\hline Variables & Values \\
\hline \multicolumn{2}{|c|}{ Sociodemographic characteristics } \\
\hline Age $>55$ years, $\mathrm{n}(\%)$ & $132(50.6)$ \\
\hline Age, years $($ Mean $\pm S D)$ & $55.6 \pm 11.8$ \\
\hline Male, n (\%) & $167(64.0)$ \\
\hline Employed, n (\%) & $96(36.8)$ \\
\hline Medical insurance, n (\%) & $43(16.5)$ \\
\hline \multicolumn{2}{|c|}{ Cerebrovascular risk factors } \\
\hline HTA, n (\%) & $191(73.2)$ \\
\hline Obesity, n (\%) & $112(42.9)$ \\
\hline Diabetes mellitus, n (\%) & $102(39.1)$ \\
\hline Alcohol consumption, n (\%) & $83(31.8)$ \\
\hline Smoking, n (\%) & $38(14.6)$ \\
\hline Previous stroke, $\mathrm{n}(\%)$ & $24(9.2)$ \\
\hline Sleep apnoea syndrome, n (\%) & $12(4.6)$ \\
\hline Dyslipidemia, n (\%) & $12(4.6)$ \\
\hline Cardiopathy, n (\%) & $9(3.5)$ \\
\hline HIV seropositive, n (\%) & $9(3.5)$ \\
\hline Migraine, n (\%) & $7(2.7)$ \\
\hline
\end{tabular}


Table 2. Clinical profile of spontaneous intracerebral haemorrhage patients.

\begin{tabular}{|c|c|}
\hline \multicolumn{2}{|l|}{ Delay in seeking medical care and CT scan confirmation } \\
\hline $\begin{array}{c}\text { Delay in initial consultation at any health facility from } \\
\text { onset of stroke symptoms (in hours) }\end{array}$ & $16.70 \pm 41.88$ \\
\hline Delay in consultation at the DGH from onset of stroke symptoms (in hours) & $52.72 \pm 84.09$ \\
\hline Delay in obtaining CT scan results from time of stroke symptoms (in hours) & $47.20 \pm 64.82$ \\
\hline \multicolumn{2}{|l|}{ Clinical parameters } \\
\hline $\mathrm{SBP}, \mathrm{mmHg}($ mean $\pm \mathrm{SD})$ & $179.3 \pm 36.5$ \\
\hline SBP > $140 \mathrm{mmHg}, \mathrm{n}(\%)$ & $222(85.1)$ \\
\hline $\mathrm{DBP}, \mathrm{mmHg}($ mean $\pm \mathrm{SD})$ & $108.1 \pm 23.6$ \\
\hline $\mathrm{DBP}>90 \mathrm{mmHg}, \mathrm{n}(\%)$ & $205(78.5)$ \\
\hline $\mathrm{CFG}, \mathrm{g} / \mathrm{L}($ mean $\pm \mathrm{SD})$ & $1.46 \pm 0.72$ \\
\hline CFG > $1.4 \mathrm{~g} / \mathrm{L}, \mathrm{n}(\%)$ & $96(36.8)$ \\
\hline Glasgow coma score (mean $\pm \mathrm{SD})$ & $10.9 \pm 3.7$ \\
\hline \multicolumn{2}{|l|}{ Site of haemorrhage on imagery } \\
\hline Basal ganglia hematoma, n (\%) & $222(85.1)$ \\
\hline Lobar hematoma, $\mathrm{n}(\%)$ & $28(10.7)$ \\
\hline Brainstem hematoma, $\mathrm{n}(\%)$ & $7(2.7)$ \\
\hline Cerebellar hematoma, n (\%) & $4(1.5)$ \\
\hline \multicolumn{2}{|l|}{ Associated anomalies on imagery } \\
\hline Intraventricular haemorrhage & $82(31.4)$ \\
\hline Mass effect, $\mathrm{n}(\%)$ & $67(25.7)$ \\
\hline Cerebral oedema, n (\%) & $23(8.8)$ \\
\hline Brain coning, $\mathrm{n}(\%)$ & $13(5.0)$ \\
\hline \multicolumn{2}{|l|}{ Etiological mechanism of ICH } \\
\hline Hypertension & $150(57.5)$ \\
\hline Confirmed arterio-venous malformation & $11(4.2)$ \\
\hline Amyloid angiopathy & $2(0.8)$ \\
\hline Undetermined & $98(37.6)$ \\
\hline
\end{tabular}

SBP: Systolic blood pressure, DBP: Diastolic blood pressure, CFG: Capillary fasting glucose.

Of the 261 patients admitted with SICH, 222 (85.1\%) and 205 (78.5\%) had systolic blood pressure (SBP) $>140 \mathrm{mmHg}$ and diastolic blood pressure (DBP) > $90 \mathrm{mmHg}$ respectively upon admission while capillary glucose $>1.4 \mathrm{~g} / \mathrm{L}$ was seen in 96 patients (36.8\%). On arrival in the Neurological Unit, 79 patients $(30.3 \%)$ with SICH had a Glasgow coma score (GCS) $\leq 8$ and were admitted at the ICU (Table 2).

With the use of CT scan, the site of haemorrhage was localised as shown in Table 1. The most frequent site of haemorrhage amongst patients with SICH 
was basal ganglia hematoma $(85.1 \%)$ while cerebellar hematoma was the least site of haemorrhage involved (1.5\%). Intraventricular blood effusion was seen in 82 patients (31.4\%) with SICH, mass effect in 67 patients $(25.7 \%)$, cerebral oedema in 23 patients $(8.8 \%)$ and brain coning occurred in 13 patients (5.0\%). This is shown in Table 2.

\section{Aetiology of $\mathrm{ICH}$ and in-hospital complication}

The most frequent aetiology of SICH in this study was hypertension (57.5\%). Chest infections $(30.7 \%)$ was the most frequent in-hospital complication seen in patients with $\mathrm{ICH}$ while pressure ulcers were the least predominant complication as seen in 6 patients $(2.3 \%)$.

Length of hospitalisation ( $\mathrm{LOH})$, functional recovery, stroke severity and mortality on admission

The mean $\mathrm{LOH}$ was $9.0 \pm 7.7$ days with range of 1 to 51 days. On arrival 81 (31.0\%) and $92(35.3 \%)$ patients with SICH had severe and very severe strokes using the NIHSS. The 24-hour post-stroke mortality rate was 9.6\% (25/261) while intra-hospital mortality rate was $39.9 \%$ (104/261). This is shown in Table 3. The most predominant cause of death amongst patients with $\mathrm{SICH}$ was severe sepsis (32.7\%) (Table 3).

Functional recovery and case fatality after stroke admission

Using the mRS, $67 \mathrm{SICH}$ survivors (61.5\%) had a poor functional outcome at month 1 while 47 ICH survivors had a poor functional outcome at month 3 after stroke onset. During 6 months of regular follow up after stroke onset, the case fatality at month 1 was $46 \%(120 / 261)$ and $59.8 \%(156 / 261)$ at month 3 (Table 3) while the overall 6 months post case fatality was $63.2 \%(165 / 261)$. Figure 2 shows the survival analysis amongst patients with $\mathrm{SICH}$.

Factors associated with in-hospital mortality amongst patients with SICH

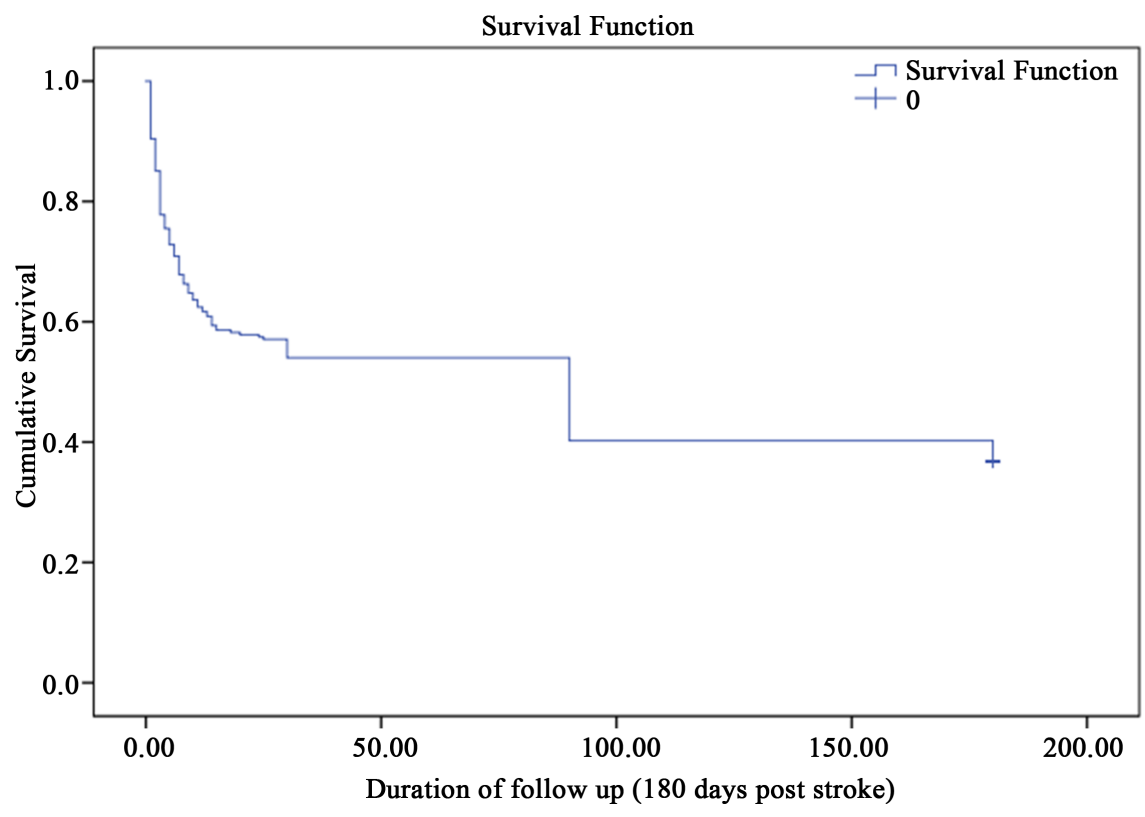

Figure 2. Survival curve of patients with SICH with a median survival period of 90 days. 
Table 3. In-hospital and 6 months outcome amongst SICH.

\begin{tabular}{|c|c|}
\hline \multicolumn{2}{|c|}{ In-hospital complications } \\
\hline Chest infection & $80(30.7)$ \\
\hline Urinary tract infection & $63(24.1)$ \\
\hline Deglutition problems & $57(21.8)$ \\
\hline Epilepsy/seizures & $29(11.1)$ \\
\hline Malaria & $23(8.8)$ \\
\hline Desaturation & $23(8.8)$ \\
\hline Chest and urinary tract co-infection & $12(4.6)$ \\
\hline Pressure ulcers & $6(2.3)$ \\
\hline \multicolumn{2}{|c|}{ Outcome on admission } \\
\hline Duration of hospital stay (days) & $9.0 \pm 7.7$ \\
\hline Rankin score $\leq 2$ & $24(9.2)$ \\
\hline Rankin score $>2$ & $237(90.8)$ \\
\hline NIHSS $<5$ & $28(10.7)$ \\
\hline NIHSS 5 - 14 & $60(23.0)$ \\
\hline NIHSS 15 - 24 & $81(31.0)$ \\
\hline NIHSS $>24$ & $92(35.3)$ \\
\hline 24-hour post-stroke mortality & $25(9.6)$ \\
\hline In hospital mortality & $104(39.9)$ \\
\hline \multicolumn{2}{|c|}{ Cause of death } \\
\hline Sepsis & $34(32.7)$ \\
\hline Multiple organ failure & $24(23.1)$ \\
\hline Coning/cerebral herniation & $17(16.3)$ \\
\hline Renal failure & $2(1.9)$ \\
\hline Undetermined & $27(26.0)$ \\
\hline \multicolumn{2}{|c|}{ Outcome within 6 months post stroke } \\
\hline Rankin score $>2$ at $\mathrm{M} 1$ & $67(61.5)$ \\
\hline Rankin score $>2$ at $\mathrm{M} 3$ & $47(50.0)$ \\
\hline Case fatality at M1 & $120(46.0)$ \\
\hline Case fatality at M3 & $156(59.8)$ \\
\hline Case fatality at M6 & $165(63.2)$ \\
\hline
\end{tabular}

On univariate analysis, the following factors were associated with in-hospital mortality after stroke onset, as seen in Table 4; alcohol abuse, GCS $<9$, NIHSS 15 - 24, NIHSS $>24$, hyperglycemia, hyperthermia, haemoglobin levels $<10 \mathrm{~g} / \mathrm{dl}$, leucopenia, leucocytosis, hypernatremia, hypokalemia, hyperkalemia, elevated CRP and elevated urea levels. On multivariate analysis, GCS $<9$ [OR $(95 \% \mathrm{CI})=$ 3.538 (1.086 - 11.526), $\mathrm{p}=0.036]$ and NIHSS $15-24[\mathrm{OR}(95 \% \mathrm{CI})=7.498$ 
Table 4. Factors associated with in hospital mortality amongst patients with SICH.

\begin{tabular}{ccccc}
\hline Variables & Alive & Dead & Unadjusted OR (95\% CI) & p-value \\
\hline Alcohol abuse & $60(74.1 \%)$ & $21(25.9 \%)$ & $0.477(0.295-0.771)$ & 0.003 \\
GCS $<9$ & $15(19 \%)$ & $64(81 \%)$ & $0.065(0.033-0.126)$ & $<0.001$ \\
NIHSS score $15-24$ & $40(60.6 \%)$ & $26(39.4 \%)$ & $4.680(1.110-19.737)$ & 0.036 \\
NIHSS score $\geq 25$ & $25(33.3 \%)$ & $50(66.7 \%)$ & $12.441(3.019-51.262)$ & $<0.001$ \\
Glycemia $>1.4 \mathrm{~g} / \mathrm{dl}$ & $59(51.3 \%)$ & $56(48.7 \%)$ & $1.813(1.220-2.694)$ & 0.003 \\
Temperature $>37.5^{\circ} \mathrm{C}$ & $47(47.5 \%)$ & $52(52.5 \%)$ & $2.017(1.372-2.966)$ & $<0.001$ \\
Hemoglobin $\leq 10 \mathrm{~g} / \mathrm{dl}$ & $5(35.7 \%)$ & $9(64.3 \%)$ & $0.457(0.229-0.912)$ & 0.026 \\
WBC $<4000 / \mathrm{mm}{ }^{3}$ & $74(77.9 \%)$ & $21(22.1 \%)$ & $0.548(0.319-0.939)$ & 0.029 \\
WBC $>10,000 / \mathrm{mm}{ }^{3}$ & $19(38 \%)$ & $31(62 \%)$ & $1.973(1.216-3.202)$ & 0.006 \\
Sodium $>145 \mathrm{mEq} / \mathrm{L}$ & $16(34 \%)$ & $31(66 \%)$ & $2.863(1.833-4.471)$ & $<0.001$ \\
Potassium $<3.5 \mathrm{mEq} / \mathrm{L}$ & $58(55.2 \%)$ & $47(44.8 \%)$ & $1.779(1.130-2.802)$ & 0.013 \\
Potassium $>5.5 \mathrm{mEq} / \mathrm{L}$ & $9(34.6 \%)$ & $17(65.4 \%)$ & $5.395(2.944-9.884)$ & $<0.001$ \\
CRP $>6 \mathrm{mg} / \mathrm{L}$ & $52(61.9 \%)$ & $32(38.1 \%)$ & $1.999(1.005-3.975)$ & 0.048 \\
Urea $>0.45 \mathrm{~g} / \mathrm{L}$ & $40(48.2 \%)$ & $43(51.8 \%)$ & $1.800(1.199-2.702)$ & 0.005 \\
\hline
\end{tabular}

(1.306 - 43.029), $\mathrm{p}=0.024]$ were seen to independent predictors of in-hospital mortality after stroke.

\section{Predictors of poor functional outcome amongst patients with ICH}

On multivariate analysis, mass effect $[\mathrm{OR}(95 \% \mathrm{CI})=3.563(1.217-10.432)$, p $=0.020]$ and hyperthermia $[\mathrm{OR}(95 \% \mathrm{CI})=4.645(1.341-16.085), \mathrm{p}=0.015]$ were seen to independent predictors of poor functional recovery after stroke (Table 5).

\section{Discussion}

In this study, the prevalence of SICH was $37 \%$ with male predominance $(64.0 \%)$ and mean age of $55.6 \pm 11.8$ years and there was no significant gender difference in age. The prevalence of haemorrhagic stroke in a three years prospective study in Cameroon was $48 \%$ with a mean age (SD) of 58.66 (13.6) years and male predominance of $68.1 \%$ [10]. This is slightly higher because of haemorrhagic stroke included ICH, subarachnoid haemorrhage and cerebral micro-bleeds only visualized on magnetic resonance imaging (MRI). This is slightly similar to the prevalence of $34.0 \%$ amongst with ICH patients aged 61 - 70 years with male predominance of $54.0 \%$ as reported by Agarwal et al. in India [12] while Desalu et al., reported a prevalence of haemorrhagic stroke of $34.7 \%$ in Nigeria [13]. The prevalence of ICH remains unchanged and usually occurs most predominantly amongst patients aged 51 - 60 and 61 - 70 years. The male predominance seen in this study is comparable to that reported most epidemiological studies [14] [15]. Stroke is more prevalent amongst the males than females because other CVRFs such as smoking and alcohol abuse are more frequent amongst the 
Table 5. Independent predictors of in hospital mortality amongst patients with SICH.

\begin{tabular}{ccc}
\hline Predictors & Adjusted OR $(95 \% \mathrm{CI})$ & p-value \\
\hline GCS $<9$ & $3.538(1.086-11.526)$ & 0.036 \\
NIHSS $[15-24]$ & $7.498(1.306-43.029)$ & 0.024 \\
\hline
\end{tabular}

men. Furthermore, the protective effect of oestrogen on the cerebral circulation reduces the risk of stroke amongst females [16].

Hypertension (HTN) was the most frequent CVRFs (73.2\%) amongst patients with SICH. According to O'Donnell et al., in 2010, 73.6\% of patients had self-reported history of HTN or BP > 160/90 mmHg [7]. Globally, several epidemiological studies have reported that HTN is the most predominant CVRFs [12] [13] [17] [18]. Hypertension is the most important risk factor for spontaneous ICH, and the contribution of hypertension is greater for deep SICH than for lobar SICH [19] [20].

On admission, deep coma occurred in $30.3 \%$, poor functional outcome using mRS occurred in $90.8 \%$ and severe to very severe stroke occurred in $66.3 \%$ of $\mathrm{SICH}$ patients. Conversely, Agarwal et al., reported that the most common presenting symptom was altered sensorium present in $58 \%$ of SICH patients while Adeleye showed that $70 \%$ of SICH patients were in a severe clinical state, $57.2 \%$ comatose; and with hemiparesis, headache, vomiting, and aphasia being the main clinical symptoms [12] [17].

The basal ganglia was the most frequent site of haemorrhage seen on imaging (85.1\%) while intraventricular blood effusion, mass effect, cerebral oedema and coning (herniation) occurred in $31.4 \%, 25.7 \%, 8.8 \%$ and $5.0 \%$ respectively. Similarly, Safatli et al., in 2016 reported that the most common hematoma location was the basal ganglia (43.9\%) [21]. According to Adeleye et al., spontaneous SICH was supratentorial on brain CT in $90.5 \%$, ganglionic in $50.8 \%$, and thalamic in $58.3 \%$ of the latter [17]. And the bleed had CT evidence of mass effect and intraventricular extension (IVH) in more than half [17]. In a population-based, prospective inception cohort study of ICH, 60 (47\%) adults had non lobar spontaneous primary first ever ICH (59 single non lobar and 1 multiple non lobar) and 68 (53\%) had lobar ICH (61 singlelobar, 4 single lobar extending to non lobar regions, 2 multiple lobar and non lobar, and 1 multiple lobar) [22]. Ezeala Adikaibe et al., in Enugu, Nigeria showed that the frequency of lobar, deep cerebral, brain stem and cerebellar hemorrhages was $46.8 \%, 44.6 \%, 3.6 \%$ and $2.2 \%$, respectively [23]. Smajlović et al., in 2008 showed that the most frequent localization of ICH was multilobar (38\%), internal capsule/basal ganglia region (36\%) and lobar (17\%) [24].

Hypertension (57.5\%) was the most predominant aetiology of $\mathrm{ICH}$. ICH is usually caused by ruptured vessels that are degenerated due to long-standing hypertension which provokes lipohyalinosis of small deep artery of the brain [25].

The mean LOH was $9.0 \pm 7.7$ days and chest infections (30.7\%) were the most 
frequent complication that occurred during admission. The average hospital stay does not depend on the type of ictus, in the sense of whether it is ischaemic or haemorrhagic, but on its severity which mainly depends on the size of the lesion [26]. The longer duration of hospital admission was due to the occurrence of multiple complications as shown in Table 4.

The case fatality rate after 24 hours (day 1), during admission, at month 1 , month 3 and month 6 was 9.6\%, 39.9\%, 46.0\%, 59.8\% and 63.2\% respectively. In Norway, the proportion of fatal outcome in retrospective analysis of data at 1 week was $22.1 \%$, at 3 months $39.2 \%$, and at 12 months $44.9 \%$ [27]. Safatli et al., in 2016 reports a 30 -day mortality in patients with SICH of $25.15 \%$ [21]. The case fatality rates reported in most studies are lower than what is seen in our context due to the time delay from onset of stroke symptoms and arrival at the stroke unit. The mean time delay in consultation at the stroke unit of $52.72 \pm$ 84.09 hours in this study, without proper antihypertensive control allows time for the hematoma volume to increase and extend to the ventricles.

On multivariate analysis, GCS $<9$ and NIHSS between $15-24$ were independent predictors of mortality while mass effect and temperature $>37^{\circ} \mathrm{C}$ were independent predictors of poor functional recovery after stroke. According to Rathor et al., the mean GCS score was significantly higher among survivors [14]. The prognostic value of low GCS and high NIHSS scores amongst SICH patients in others study probably reflects the mass effects of hematoma collection, intraventricular hemorrhage and intraventricular hematoma extension. In this study, $81 \%$ of ICH patients with GCS $<9$ died in the acute phase of stroke (Table 4). Sepsis was the most common complications on admission and the most predominant cause of death (32.7\%). This explains why the SICH patients with fever had poor functional outcome during admission as most septic patients present with fever. Fever increases mortality, worsens stroke severity and increases the intensive care length of stay and hospital length of stay [28]. A recent retrospective population-based study in Norway showed that predictors of severe disability or death were use of oral antithrombotic drugs, functional disability prior to SICH, low GCS on admission, larger hematoma volume, and intraventricular hematoma extension [27]. Smajlović et al., showed that mortality and good outcome at 1 month, is related to the localization of bleeding and that age, stroke severity, multilobar haemorrhage and intraventricular haemorrhage were factors independently associated with mortality [24]. Newly diagnosed diabetes mellitus was independently associated with 1-year poor functional outcome but showed no significant association with 1-year death and stroke recurrence [29]. Koivunen et al., in a follow up study of SICH patients aged 16 - 49 years showed that unfavorable functional outcome emerged in $49 \%$ and age, initial stroke severity and intraventricular blood effusion were associated with unfavourable functional outcome while male sex and diabetes were associated with increased mortality after adjusting for age and intraventricular hematoma extension [30] (Table 6). 
Table 6. Independent predictors of poor functional outcome amongst patients with ICH.

\begin{tabular}{ccc}
\hline Variables & Adjusted OR (95\% CI) & p value \\
\hline Age $\geq 65$ years & $3.980(0.601-26.349)$ & 0.152 \\
Profession & $0.659(0.132-3.286)$ & 0.611 \\
Alcohol abuse & $0.317(0.100-1.010)$ & 0.052 \\
Mass effect & $3.563(1.217-10.432)$ & 0.020 \\
Furation of hospitalisation $>7$ days & $3.011(0.905-10.018)$ & 0.072 \\
Female & $0.510(0.133-1.958)$ & 0.327 \\
Temperature $>37.5^{\circ} \mathrm{C}$ & $\mathbf{4 . 6 4 5 ( 1 . 3 4 1 - 1 6 . 0 8 8 )}$ & $\mathbf{0 . 0 1 5}$ \\
Urea $>0.45 \mathrm{~g} / \mathrm{L}$ & $1.720(0.503-5.886)$ & 0.387 \\
NIHSS $>14$ & $1.509(0.495-4.597)$ & 0.469 \\
\hline
\end{tabular}

The major limitation of this study is the absence of information on the volume of the hematoma which is a probable predictor of poor stroke outcomes.

\section{Conclusion}

About one third of stroke patients were haemorrhagic. Hypertension is the leading CVRF and aetiology of SICH. About 1 over 2 patients with SICH would die within 6 months. Therefore, primary prevention and optimal management of SICH victims are crucial in the reduction of SICH related mortality and morbidity.

\section{Conflicts of Interest}

The authors declare no conflicts of interest regarding the publication of this paper.

\section{References}

[1] Murray, C.J.L. and Lopez, A.D. (1996) The Global Burden of Disease: A Comprehensive Assessment of Mortality and Disability from Diseases, Injuries, and Risk Factors in 1990 and Projected to 2020. Harvard School of Public Health, Cambridge.

[2] Mathers, C., Fat, D.M., Boerma, J.T. and World Health Organization (2008) The Global Burden of Disease, 2004 Update. World Health Organization, Geneva.

[3] Mukherjee, D. and Patil, C.G. (2011) Epidemiology and the Global Burden of Stroke. World Neurosurgery, 76, S85-S90. https://doi.org/10.1016/j.wneu.2011.07.023

[4] Deresse, B. and Shaweno, D. (2015) Epidemiology and In-Hospital Outcome of Stroke in South Ethiopia. Journal of the Neurological Sciences, 355, 138-142. https://doi.org/10.1016/j.jns.2015.06.001

[5] Berkowitz, A.L. (2015) Stroke and the Noncommunicable Diseases, a Global Burden in Need of Global Advocacy. Neurology, 84, 2183-2184. https://doi.org/10.1212/WNL.0000000000001618

[6] Feigin, V.L., Lawes, C.M., Bennett, D.A. and Barker-Collo, S.L. and Parag, V. (2009) 
Worldwide Stroke Incidence and Early Case Fatality Reported in 56 PopulationBased Studies, a Systematic Review. Lancet Neurology, 8, 355-369. https://doi.org/10.1016/S1474-4422(09)70025-0

[7] O’Donnell, M.J., Xavier, D., Liu, L., Zhang, H., Chin, S.L., Rao-Melacini, P., et al. (2010) Risk Factors for Ischaemic and Intracerebral Haemorrhagic Stroke in 22 Countries (The Interstroke Study), a Case-Control Study. The Lancet, 376, 112-123. https://doi.org/10.1016/S0140-6736(10)60834-3

[8] Kaae, A.K., Skyhøj, O.T., Christian, D. and Peter, K.L. (2009) Hemorrhagic and Ischemic Strokes Compared: Stroke Severity, Mortality, and Risk Factors. Stroke, 40, 2068-2072. https://doi.org/10.1161/STROKEAHA.108.540112

[9] Lekoubou, A., Nkoke, C., Dzudie, A. and Kengne, A.P. (2015) Stroke Admission and Case-Fatality in an Urban Medical Unit in Sub-Saharan Africa, a Fourteen Year Trend Study from 1999 to 2012. Journal of the Neurological Sciences, 350, 24-32. https://doi.org/10.1016/j.jns.2015.02.002

[10] Mapoure, Y.N., Kuate, C., Tchaleu, C.B., Ngahane, H.B.M., Mounjouopou, G.N., Ba, H., et al. (2014) Stroke Epidemiology in Douala, Three Years Prospective Study in a Teaching Hospital in Cameroon. World Journal of Neuroscience, 4, 406-414. https://doi.org/10.4236/wjns.2014.45044

[11] Poon, M.T.C., Bell, S.M. and Al-Shahi, S.R. (2015) Epidemiology of Intracerebral Haemorrhage. In: Toyoda, K., Anderson, C.S. and Mayer, S.A., Eds., New Insights in Intracerebral Hemorrhage, Frontiers of Neurology and Neuroscience, Karger, Basel, 1-12. https://www.karger.com/Article/Abstract/437109 https://doi.org/10.1159/000437109

[12] Agarwal, R.K., Kulshreshtha, D., Maurya, P.K., Singh, A.K. and Thacker, A.K. (2016) Clinical Features and Predictors of in Hospital Mortality in Patients with Intra Cerebral Haemorrhage. International Journal of Research in Medical Sciences, 4 , 836-840. https://doi.org/10.18203/2320-6012.ijrms20160528

[13] Desalu, O., Wahab, K., Fawale, B., Olarenwaju, T., Busari, O., Adekoya, A., et al. (2011) A Review of Stroke Admissions at a Tertiary Hospital in Rural Southwestern Nigeria. Annals of African Medicine, 10, 80-85. https://doi.org/10.4103/1596-3519.82061

[14] Rathor, M.Y., Rani, M.F.A., Jamalludin, A.R., Amran, M., Shahrin, T.C.A. and Shah, A. (2012) Prediction of Functional Outcome in Patients with Primary Intracerebral Hemorrhage by Clinical-Computed Tomographic Correlations. Journal of Research in Medical Sciences, 17, 1056-1062.

[15] Barber, M., Roditi, G., Stott, D.J. and Langhorne, P. (2004) Poor Outcome in Primary Intracerebral Haemorrhage, Results of a Matched Comparison. Postgraduate Medical Journal, 80, 89-92. https://doi.org/10.1136/pmj.2003.010967

[16] Krause, D.N., Duckles, S.P. and Pelligrino, D.A. (2006) Influence of Sex Steroid Hormones on Cerebrovascular Function. Journal of Applied Physiology, 101, 12521261. https://doi.org/10.1152/japplphysiol.01095.2005

[17] Adeleye, A.O., Osazuwa, U.A. and Ogbole, G.I. (2015) The Clinical Epidemiology of Spontaneous ICH in a Sub-Sahara African Country in the CT Scan Era: A Neurosurgical In-Hospital Cross-Sectional Survey. Frontiers in Neurology, 6, 169.

https://www.frontiersin.org/articles/10.3389/fneur.2015.00169/full https://doi.org/10.3389/fneur.2015.00169

[18] Sturgeon, J.D., Folsom, A.R., Longstreth, W.T., Shahar, E., Rosamond, W.D. and Cushman, M. (2007) Risk Factors for Intracerebral Hemorrhage in a Pooled Prospective Study. Stroke, 38, 2718-2725. 
https://doi.org/10.1161/STROKEAHA.107.487090

[19] Zia, E., Hedblad, B., Pessah-Rasmussen, H., Berglund, G., Janzon, L. and Engström, G. (2007) Blood Pressure in Relation to the Incidence of Cerebral Infarction and Intracerebral Hemorrhage. Hypertensive Hemorrhage, Debated Nomenclature Is Still Relevant. Stroke, 38, 2681-2685. https://doi.org/10.1161/STROKEAHA.106.479725

[20] Martini, S.R., Flaherty, M.L., Brown, W.M., Haverbusch, M., Comeau, M.E., Sauerbeck, L.R., et al. (2012) Risk Factors for Intracerebral Hemorrhage Differ According to Hemorrhage Location. Neurology, 79, 2275-2282. https://doi.org/10.1212/WNL.0b013e318276896f

[21] Safatli, D.A., Günther, A., Schlattmann, P., Schwarz, F., Kalff, R. and Ewald, C. (2016) Predictors of 30-Day Mortality in Patients with Spontaneous Primary Intracerebral Hemorrhage. Surgical Neurology International, 7, S510-S517. https://doi.org/10.4103/2152-7806.187493

[22] Samarasekera, N., Fonville, A., Lerpiniere, C., Farrall, A.J., Wardlaw, J.M., White, P.M., et al. (2015) Influence of Intracerebral Hemorrhage Location on Incidence, Characteristics, and Outcome, Population-Based Study. Stroke, 46, 361-368.

[23] Ezeala-Adikaibe, B.A. and Ohaegbulam, S.C. (2016) Pattern and Location of Intracerebral Hemorrhage in Enugu, South-East Nigeria, a Review of 139 Cases. Nigerian Journal of Clinical Practice, 19, 332-325. https://doi.org/10.4103/1119-3077.179295

[24] Smajlović, D., Salihović, D., Ibrahimagić, O.Ć., Sinanović, O. and Vidović, M. (2008) Analysis of Risk Factors, Localization and 30-Day Prognosis of Intracerebral Hemorrhage. Bosnian Journal of Basic Medical Sciences, 8, 121-125. https://doi.org/10.17305/bjbms.2008.2964

[25] An, S.J., Kim, T.J. and Yoon, B.-W. (2017) Epidemiology, Risk Factors, and Clinical Features of Intracerebral Hemorrhage, an Update. Journal of Stroke, 19, 3-10. https://doi.org/10.5853/jos.2016.00864

[26] Láinez, J.M., Pareja, A. and Santonja, J.M. (1997) Hospital Stay after Cerebral Hemorrhage. Revue Neurologique, 25, 1121-1125.

[27] Øie, L.R., Madsbu, M.A., Solheim, O., Jakola, A.S., Giannadakis, C., Vorhaug, A., et al. (2018) Functional Outcome and Survival Following Spontaneous Intracerebral Hemorrhage: A Retrospective Population-Based Study. Brain and Behavior, 8, e01113. https://www.ncbi.nlm.nih.gov/pmc/articles/PMC6192392/ https://doi.org/10.1002/brb3.1113

[28] Greer, D.M., Funk, S.E., Reaven, N.L., Ouzounelli, M. and Uman G.C. (2008) Impact of Fever on Outcome in Patients with Stroke and Neurologic Injury. Stroke, 39, 3029-3035. https://doi.org/10.1161/STROKEAHA.108.521583

[29] Zhang, X., Jing, J., Zheng, H., Jia, Q., Zhao, X., Liu, L., et al. (2018) Prognosis of Intracerebral Hemorrhage with Newly Diagnosed Diabetes Mellitus According to Hemoglobin A1c Criteria. Journal of Stroke and Cerebrovascular Diseases, 27, 1127-1133.

[30] Koivunen, R.-J., Tatlisumak, T., Satopää, J., Niemelä, M. and Putaala, J. (2015) Intracerebral Hemorrhage at Young Age: Long-Term Prognosis. European Journal of Neurology, 22, 1029-1037. https://doi.org/10.1111/ene.12704 